\title{
DOZENT SLEIMS HERCBERGS - GRÜNDER UND ERSTER LEITER DES LEHRSTUHLES FÜR THERAPEUTISCHE STOMATOLOGIE AN DER STAATLICHEN UNIVERSITÄT LETTLAND
}

Einen ausführlichen Bericht über den Wiederaufbau der Lettischen Hochschule für Medizin in den Nachkriegsjahren, über die Menschen, die dabei mitgewirkt haben, und die Bedeutung ihrer Tätigkeit hat der Medizinhistoriker A. Vīksna veröffentlicht. Angaben über den Dozenten S. Hercbergs sind sehr lakonisch und umfassen einen Teil im Satz, worin es heißt, daß "therapeutische Stomatologie vom Dozenten Sleims Hercbergs betreut wurde" [1]. Tatsächlich war es für den Medizinhistoriker recht schwierig, weitgehende Informationen über diesen Menschen zu ermitteln, denn sein Name war fast völlig aus den amtlichen Unterlagen verschwunden (vielleicht wäre eher zu sagen - bewußt gestrichen).

Anfang der fünfziger Jahre wurde in der damaligen Sowjetunion das sogenannte Verfahren "Vergifterärzte" in Bezug auf jüdische Ärzte durchgeführt. Dadurch haben die Hochschulen für Medizin in Sibirien bedeutend ihr Ansehen erhöht, denn namhafte Fachleute und Wissenschaftler vom Bereich der Medizin wurden dorthin verschickt [2]. Zum Glück ist es nicht mit S. Hercbergs geschehen. Wahrscheinlich aus dem Grund, daß er große Autorität auf den medizinischen, wissenschaftlichen und pädagogischen Gebieten war. Er genoß eine beachtliche Popularität nicht nur unter Medizinern, sondern auch unter den Ausführern dieser Aktion. Innerhalb eines Tages hatte der Lehrstuhlleiter seine Tätigkeit an der Hochschule aufzugeben. Er sollte eigenhändig Anträge für die Überweisung als Abteilungsleiter der Zahnbehandlung an der 10. Poliklinik der Stadt Riga schreiben: "Auf eigenem Wunsch kündige ich meine Stelle an der Staatlichen Universität Lettlands" (Anordnung des Universitätshauptamtes Nr. 133 / VK vom 19. Februar 1951) [3].

Damals wurde verordnet, die so genannten Personalakten von allen ehemaligen Hochschullehrkräften in das Archiv der entsprechenden Hochschule aufzunehmen, d.h. Zusammenfassung von Unterlagen betreffend der Ausbildung, Arbeit, Auszeichnungen, des Gelehrtengrades, Postens usw. 
Es kann angenommen werden, daß die Archive der Akademie für Medizin Lettlands und der Lettischen Universität ordentlich geführt waren, dennoch fehlt hier die Personalakte des Ex-Lehrstuhlleiters S. Hercbergs. In den Erläuterungen der Archivmitarbeiter heißt es, daß einzelne Akten der Lehrkräfte dem Staatssicherheitskomitee zur Verfügung gestellt wurden, möglicherweise waren die Unterlagen von S. Hercbergs darunter.

Die Namen von bedeutendsten Lehrkräften der Lettischen Universität und der Akademie für Medizin, vor allem die der Lehrstuhlleiter sind im "Lettischen Sowjetischen Konversationslexikon" enthalten. Recht ausführlich werden sie in der Anlage zum "Populären Medizinlexikon", Auflage 1985 behandelt, doch ist der Name des Dozenten S. Hercbergs in diesen Ausgaben verschwiegen worden.

Das gleiche kommt in vielen Veröffentlichungen zur Geschichte der lettischen Stomatologie vor. Aus den Gesprächen mit lettischen Stomatologen, die bis zu den fünfziger Jahren studiert haben, ist zu entnehmen, daß der Dozent S. Hercbergs als einer der Grundsteinleger der lettischen Stomatologiegeschichte besondere Hochachtung genossen hat, als einer der viel zur Entwicklung der Stomatologie beigetragen hat, ein hervorragender Pädagoge und erfahrener Arzt mit weitreichenden Kenntnissen bei der Behandlung der Mundhöhlenerkrankungen war.

Über die Einstellung seiner Arbeit an der Lettischen Universität werden Vermutungen geäußert, - Zusammenarbeit mit ausländischen Kollegen, die als Todsünde empfunden wurde, Beherrschung von mehreren Sprachen, Rettung vor den Todeslagern der Nazis, was den Überwachern der Hochschulen verdächtig vorkommen mochte.

Unsere Überzeugung davon, daß der Name des Dozenten S. Hercbergs einen bedeutenden Platz in der Geschichte der lettischen Stomatologie verdient hat, jedoch bis heute keine historischen Materialien über ihn geordnet worden sind, hat uns zur Erfassung und Zusammentragen dieser Angaben bewegt.

Der Verfasser bedankt sich bei Herrn Professor G. Pakalns, Vorsitzender des wissenschaftlichen Vereins der lettischen Stomatologen, für die zur Verfügung gestellten Archivunterlagen des Vereins, worin wir unwiderlegbare Nachweise für die historische Rolle des Wirkens von S. Hercbergs gefunden haben. Herr Professor Pakalns hat auch die Befragungsdurchführung der Kollegen bzw. Helfern des Dozenten S. Hercbergs unterstützt.

Der Bericht beruht auch auf Unterlagen, die von der Generaldirektion des Staatsarchivs betreut werden, Materialien und Veröffentlichungen des Pauls Stradins Museums für Medizingeschichte, die in Bibliotheken zu lesen sind. Die Gesamtheit der angeführten Materialien liefert einen Beweis dafür, daß Dozent S. Hercbergs eine der hervorragendsten Persönlichkeiten der lettischen Stomatologie darstellt und einen großen Beitrag zur Entfaltung der stomatologischen Wissenschaft und Praxis im Laufe der Nachkriegszeit geleistet hat. 


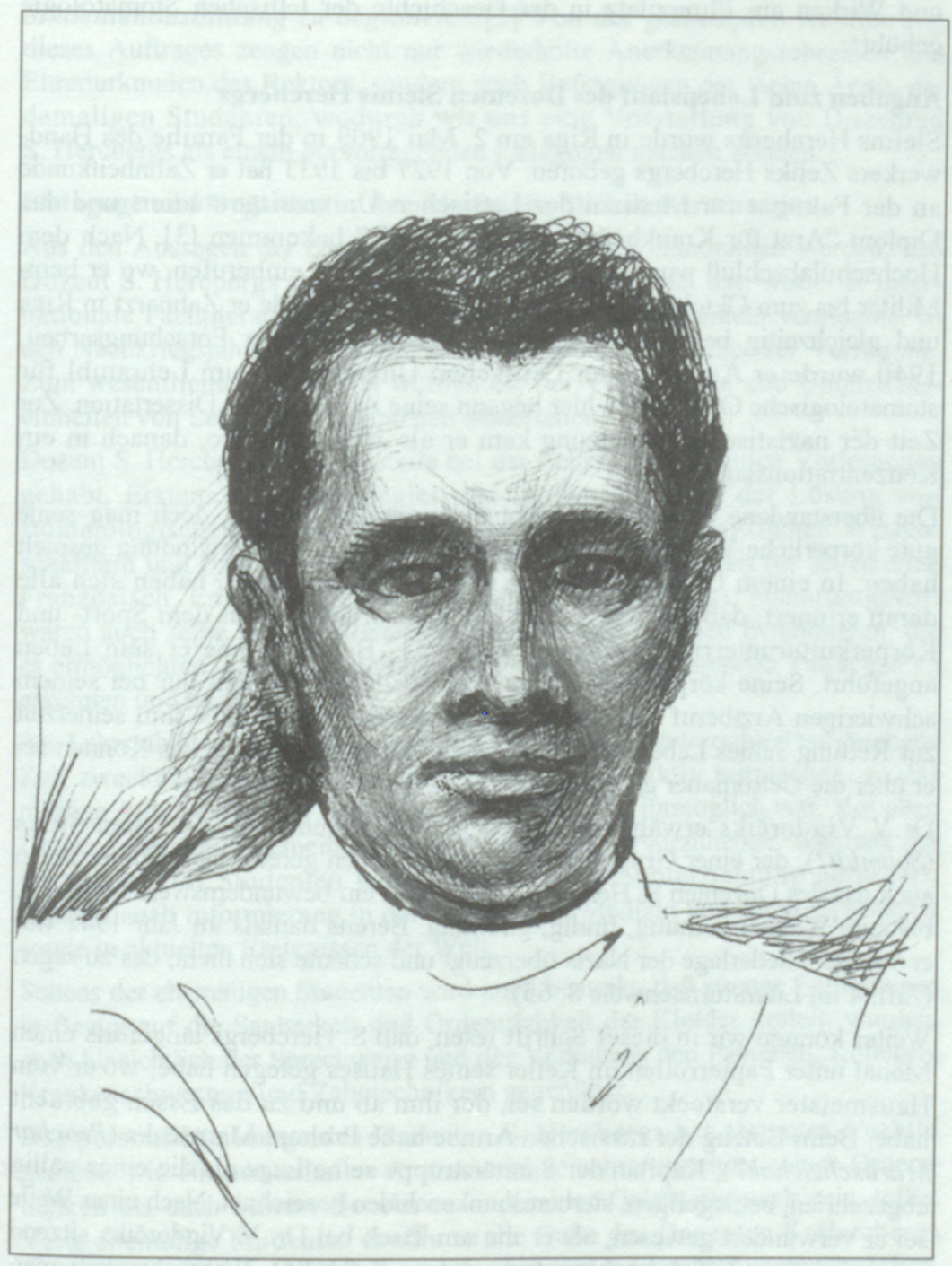

Dozent Sleims Hercbergs. Zeichnung (Autor unbekannt) 
Aus politischen Gründen wurde der Name des Dozenten S. Hercbergs verschwiegen, doch der Gerechtigkeit halber ist zu bekennen, daß seinem Leben und Wirken ein Ehrenplatz in der Geschichte der lettischen Stomatologie gebührt.

\section{Angaben zum Lebenslauf des Dozenten Sleims Hercbergs}

Sleims Hercbergs wurde in Riga am 2. Mai 1909 in der Familie des Handwerkers Zeliks Hercbergs geboren. Von 1927 bis 1933 hat er Zahnheilkunde an der Fakultät für Medizin der Lettischen Universität studiert und das Diplom "Arzt für Krankheiten der Mundhöhle" bekommen [3]. Nach dem Hochschulabschluß wurde er in die lettische Armee einberufen, wo er beim Militär bis zum Oktober 1934 gedient hat. Danach wurde er Zahnarzt in Riga und gleichzeitig beschäftigte sich mit wissenschaftlicher Forschungsarbeit. 1940 wurde er Assistent der Lettischen Universität beim Lehrstuhl für stomatologische Orthopädie, hier begann seine Arbeit an der Dissertation. Zur Zeit der nazistischen Besatzung kam er als Jude ins Getto, danach in ein Konzentrationslager.

Die überstandene Qualen sind nicht dokumentiert worden, doch mag seine gute körperliche Verfassung eine große Rolle bei ihrer Überwindung gespielt haben. In einem Gespräch mit den ehemaligen Studenten haben sich alle daran erinnert, daß Dozent S. Hercbergs ausdrücklich auf dem Sport- und Körperkulturunterricht bestanden habe. Als Beispiel habe er sein Leben angeführt. Seine körperliche Stärke und Ausdauer sei nicht nur bei seinem schwierigen Arztberuf von Bedeutung gewesen, sondern habe ihm seinerzeit zur Rettung seines Lebens verholfen, denn durch sein sportliches Können sei er über die Gettomauer gesprungen.

Dr. V. Vigdorčiks erwähnt in seinen Erinnerungen den Deutschen Šmits (Schmidt?), der einer Gruppe von Juden das Leben gerettet habe, unter ihnen auch das des Dozenten S. Hercbergs. Smits war ein bewundernswerter Mensch, bis zum Wahnsinn mutig, findig, gutmütig. Bereits damals im Jahr 1942 war er von der Niederlage der Nazis überzeugt und scheute sich nicht, das zu sagen (Ziff. 4 im Literaturnachweis S. 65).

Weiter können wir in dieser Schrift lesen, daß S. Hercbergs länger als einen Monat unter Papierrollen im Keller seines Hauses gelegen habe; wo er vom Hausmeister versteckt worden sei, der ihm ab und zu das Essen gebracht habe. Beim Einzug der russischen Armee habe Prohors Maruščaks (Prochor Maruschtschak?), Kapitän der Sanitätstruppe seine Lage als die eines vőllig abgezehrten, bettlägerigen, sterbenskranken Juden bezeichnet. Nach einer Weile sei er verwundert gewesen, als er ihn am Tisch bei Dr. V. Vigdorčiks sitzend gesehen habe (Ziff. 4 im Literaturnachweis S. 84-85). Wahrscheinlich mag auch hier seine körperliche Stählung eine Rolle gespielt haben.

Das Weitere ist mit einem Zitat aus dem Lebenslauf zu belegen: "Am 17. Oktober 1944 wurde ich von der provisorischen Leitung der Staatlichen 
Universität Lettland beauftragt, den gestörten Lehrstuhl für therapeutische Stomatologie zu organisieren, Krankenaufnahme zu ermöglichen und mit der Studentenausbildung zu beginnen. " [3] Von der glänzenden Ausführung dieses Auftrages zeugen nicht nur wiederholte Anerkennungsschreiben und Ehrenurkunden des Rektors, sondern auch Befragungen der vielen Ärzte, der damaligen Studenten, wodurch wir uns eine Vorstellung von Dozenten S. Hercbergs als einem hervorragenden Pädagogen machen können.

\section{Pädagoge und Organisator der wissenschaftlichen Forschungen}

Aus den Aussagen der ehemaligen Studenten kann entnommen werden, daß Dozent S. Hercbergs spannende Vorlesungen gehalten hat, wobei er internationale Fachliteratur jener Jahre systematisch zu behandeln vermochte. In den Nachkriegsjahren hatten die Studenten keine Lehrbücher zur Verfügung. Zum wesentlichen Lehrmittel wurden die bei Vorlesungen und Unterrichtseinheiten von Lehrkräften gebotenen Materialien.

Dozent S. Hercbergs hatte Vorteile bei der Erstellung von guten Vorlesungen gehabt. Erstens seine Wißbegier und Leidenschaft bei der Lösung von stomatologischen Fragen; zweitens Kontakte zu fortschrittlichen Wissenschaftlern und Praktikern jener Zeit; drittens Sprachfertigkeit (er beherrschte Französisch, Englisch, Deutsch, Russisch und Lettisch); bedeutungsvoll waren auch seine großen wissenschaftlichen und ärztlichen Erfahrungen, die es ermöglichten, das Hauptsächliche unter weniger bedeutenden Tatsachen zu erkennen und hervorzuheben.

Als Lehrstuhlleiter hat er strikt geachtet, daß die zum Unterrichten vorgesehene Zeit zweckmäßig genutzt wird, daß die Studenten aktiv mitmachen, so das müßige Anwesenheit der Studenten beim Unterricht unmöglich war. Vor allen Dingen wäre die Allgemeinbildung der Studenten hervorzuheben. Während der Gespräche mit Studenten wurde nicht nur Fachunterrichtung gefordert, sondern auch Informierung in der allgemeinen Medizin und sogar Geschichte sowie in aktuellen Ereignissen der Welt.

Seitens der ehemaligen Studenten wird noch bemerkt, daß strenge Forderungen in Bezug auf die Sauberkeit und Ordentlichkeit der Kleider gestellt wurden, auch hinsichtlich der Sprechweise und des Verhaltens den Patienten, Kollegen, Krankenschwestern und Zahntechnikern gegenüber.

Das persönliche Image des Dozenten S. Hercbergs hat als gutes Vorbild gedient, wie ein musterhafter Arzt sowohl seinem Aussehen, seiner Ordentlichkeit als auch seinem Benehmen und seiner Galanterie nach sein sollte. Viele ehemalige Studenten erwähnen die Gabe des Dozenten S. Hercbergs schlagfertig zu scherzen, Sachen und Erscheinungen von ihrer ungewöhnlichen, sogar witzigen Seite zu betrachten. Dabei meinen einige Ex-Studenten, das wäre ein zu besprechendes und bezweifelndes Anliegen, da Haltung dieser Art beim Unterricht kaum zugelassen werden dürfte. 
Zum Beispiel, Frühlingsprüfungen werden in einem schwülen, stickigen Raum abgenommen. Der Hauptprüfer ist Lehrstuhlleiter, als Mitprüferin beteiligt sich eine Assistentin, die am Fenster sitzt; die zu prüfende Studentin weiß nichts über die Frage. Der Prüfer bittet seine Assistentin: "Kollegin, seien Sie so lieb und öffnen Sie bitte das Fenster, es stinkt stark nach Ungewißheit." Nach so vielen Jahren erinnert sich die Ärztin mit Pein daran.

Neben der pädagogischen Tätigkeit hat Dozent S. Hercbergs viel Zeit und Mühe der Organisation und Erstellung der Methodik für die staatliche zahnärztliche Hilfe gewidmet. Dies wurde vor allem im Rahmen der vorbildlich ausgeführten Aufgaben des Sekretärs beim wissenschaftlichen Verein der lettischen Stomatologen durchgeführt. Zur sowjetischen Zeit wurden durch die Wirkung des Vereins Richtlinien der stomatologischen Angelegenheiten in Lettland bestimmt.

Hier wurden Möglichkeiten der zahnärztlichen Vorbeugungsmaßnahmen durchgesehen, die zahnärztliche Behandlung in Kindergärten und Schulen ausgewertet. Es erfolgte auch eine Auswertung der Arbeitsverfahren und Arbeitsorganisation in einzelnen Polikliniken und Zahnarztpraxen in Lettland. Nach Protokollen des Vereins zu urteilen, wurde die ganze Arbeit in Kooperation von Professor Dominiks Kalvelis, dem Vereinsvorsitzenden und Dozenten Sleims Hercbergs, dem Sekretär geleistet [5].

Dozent S. Hercbergs war anscheinend der Überzeugung, daß erfolgreiche Behandlung nicht nur von der Arbeitsorganisation, Materialien und Verfahren bestimmt wird, sondern auch in hohem Maße von der eigenen Einstellung der Patienten und ihrem Verständnis für das Anliegen. Daher wurden 1949 die populärwissenschaftlichen Abhandlungen für die Einwohnerschaft herausgegeben "Zahnfäule und seine Auswirkung auf die menschliche Gesundheit" und "Was soll die Mutter wissen, damit ihr Kind mit gesunden und starken Zähnen heranwachsen kann" [3].

Diese Ausgaben scheinen Verwirklichung vom lange gehegten Plan zu sein. Fesselnd ist das am 28. November 1940 erstellte Projekt, unterzeichnet von Prof. D. Kalvelis und Dr. S. Hercbergs, - ein Projekt zur Bekämpfung von Anomalien beim Kiefer und dem Gebiß der Kinder, woran außer den Unterzeichnern auch drei Schulzahnärztinnen mitgewirkt haben (M. Kilbloka, Z. Lācere und V. Tiltiṇa).

Dem Inhalt nach scheint es ein recht vollkommenes und interessantes Projekt zu sein, einen bedeutenden Teil stellt die Ausführung von Druckschriften und Anschauungsmitteln über die Zahnpflege und sonstige Vorbeugungsmaßnahmen für die Schulen dar [5].

Die weitere zahnärztliche Entwicklung zeugt von Ausführung zentralisierter, von oben vorgeschriebener Hinweise. Das Volkskommissariat für Gesundheitsschutz hat den wissenschaftlichen Rat mit einem stomatologischen Ausschuß gegründet. Zu seinem Vorsitzenden wurde Prof. D. Kalvelis, 
Mitglieder waren Frau I. Leja, Lehrstuhlleiterin für chirurgische Stomatologie, Herr S. Hercbergs, Lehrstuhlleiter für therapeutische Stomatologie und Herr Assistent H. Andersons (Protokoll vom 26. April 1945) [5].

Bei einer Ausschußsitzung wurde entschieden, daß Zahnärzte bei gemeinsamen Sitzungen zusammenkommen sollen, um Fragen des Einsatzes von Materialien, Arbeitsorganisation usw. zu behandeln. Ähnliche Besprechungen fanden in der Vorkriegszeit als Vereinssitzungen statt, die auf keinen Fall erwähnt werden durften, und 1946 wurde von neuem medizin-wissenschaftlicher Verein von Stomatologen der Lettischen SSR gegründet. Es sei zu bemerken, daß die im Jahre 1908 angefangene Dokumentation fortgesetzt wird, lediglich ist der Vereinsname mehrmals geändert worden. Zu der Gründergruppe gehören Prof. D. Kalvelis, J. Zbars, Kriegsarzt, später Professor in Petersburg, Dozent S. Hercbergs, Dr. V. Vigdorčiks und Dozent H. Andersons [6].

Wir können Dozenten S. Hercbergs als einen der Vereinsgründer in der Nachkriegszeit betrachten. Aktivität und Engagement charakterisieren sein Wirken als Vereinssekretär. Die unter den Vereinsmitgliedern herrschende große Hochachtung ihm gegenüber zeigt sich bei der ordentlichen Vorstandswahl am 20. Dezember 1951, als für ihn 57 von den 73 möglichen Stimmen abgegeben wurden.

$\mathrm{Zu}$ diesem Zeitpunkt wurde im Zentrum der damaligen Sowjetunion das Verfahren gegen jüdische Mediziner "Vergifterärzte" vorbereitet. Auch in Lettland spiegelte sich das Ereignis wider und die Richtlinien der Partei wurden bedingungslos durchgeführt. Im Protokoll des Vereins der Stomatologen vom 21. Februar 1952 läßt sich sehen, daß bei der Besprechung vom Vertreter des wissenschaftlichen Rates der Medizin, georgischen Kriegsarzt Arčils Mačabeli (Professor, Gründer des Instituts für Traumatologie und Orthopädie Riga) berichtet wird, daß "die am 20. Dezember 1951 durchgefilhrte Wahl im Verein der Stomatologen nicht vom wissenschaftlichen Rat der Medizin vorgesehen war. Es gibt Ungenauigkeiten im Protokoll der Stimmenzählung, ebenso war der Bestand nicht durchgearbeitet. Vom wissenschaftlichen Rat der Medizin wird eine Neuwahl vorgeschlagen" (der zitierte Protokollauszug ist aus dem Russischen übersetzt worden). Von der demobilisierten Kapitänin, russischer Zahnärztin A. Gogulina wird die Bewerberliste für den Vorstand ohne S. Hercbergs und V. Vigdorčiks vorgelesen. Die Niederschrift enthält auch einen Hinweis, daß ihre Namen von Wählern eigenhändig in mehrere Wahlzettel eingetragen wurden, was freilich zur Änderung des Wahlergebnisses nicht führen konnte, brachte jedoch Achtung diesen Menschen entgegen.

$\mathrm{Zu}$ dieser Zeit war Dozent S. Hercbergs gezwungen, seine Arbeit an der Hochschule aufzugeben und die Laufbahn eines praktizierenden Arztes als Abteilungsleiter in der Rigaer 10. Poliklinik zu beginnen. Selbstverständlich hat er seine Aufgaben glänzend erfüllt, sowohl die Ärzte als auch das Hilfspersonal haben ihn in bester Erinnerung behalten, doch sind die Kollegen der 
Meinung, daß das nicht sein richtiger Platz war, sein Herz hing an der stomatologischer Wissenschaft und sein Geist schwebte in höheren Sphären.

Parallel mit der Arbeit in der 10. Poliklinik hat er seine wissenschaftlichen Forschungen ehrenamtlich an der Akademie der Wissenschaften in der Abteilung für Biologie und Landwirtschaft fortgesetzt. Trotz der Einschränkung seiner Tätigkeit beim Vorstand hat sich Dozent S. Hercbergs aktiv an den Vereinssitzungen der Stomatologen beteiligt. Im Besprechungsprotokoll vom 18. März 1954 gibt es eine Eintragung über seine Teilnahme an Debatten zur Anwendung der Anästhesie in der Stomatologie. In die persönliche Akte beim Archiv ist wiederum eingetragen worden "Gestorben am 7. April 1954".

Mit Überzeugung kann man behaupten, daß trotz der Schwierigkeiten während des Krieges und des erlittenen Unrechts Dozent S. Hercbergs sein ganzes Leben der Entfaltung der stomatologischen Wissenschaft in Lettland gewidmet hat und daß seinem Namen verdienter Ehrenplatz in der Geschichte der lettischen Stomatologie gebührt.

Von ganzem Herzen bedanke ich mich bei den vielen ehemaligen Studenten und Kollegen des Dozenten S. Hercbergs für die bei der Befragung anvertrauten Angaben.

\section{Literaturnachweis}

1. Vīksna A. Gründung des Instituts für Medizin Riga // Latvijas Ärsts [Lettischer Arzt]. - 1997. - Nr. 11. - S. 699-709.

2. Alks D. Lettische Mediker in der Mühle der politischen Verfolgung 1940-1953. Riga: Ausgabe des Vereins von lettischen Ärzten und des nationalen Kulturvereins der lettischen Juden, [1993]. - S. 1-104.

3. Unterlagenarchiv des Personalbestandes von Riga und Jūrmala, 283 f, 1. Beschreibung 31.1.

4. Vigdorčiks V. Meine Chronik des zwanzigsten Jahrhunderts. - 1992. - Abteilung für Handschriften des Pauls-Stradins-Museum der Medizingeschichte, 42. 254 / R-31806; S. $1-130$.

5. Besprechungsprotokolle des wissenschaftlichen Vereins der lettischen Stomatologen 1908-1945, 1946-1954, Vereinsarchiv.

6. Kalvelis D., Pakalns $G$. Die Rolle des wissenschaftlichen stomatologischen Medizinvereins der Lettischen SSR bei der Entwicklung der wissenschaftlichen und praktischen Stomatologie der Republik // Stomatologijas attīstība Latvijas PSR [Entwicklung der Stomatologie in der Lettischen SSR]. - Riga, 1967. - S. 81-97.

\section{Isaak Gutermann}

Grüner Weg $28^{\mathrm{A}}$, D-51375 Leverkusen, Deutschland 